\title{
Affordances of Twitter as a pedagogical tool in education: critical analysis of doctoral research studies
}

\section{Myint Swe Khine}

Assessment and Evaluation Center, Emirates College for Advanced Education, Abu Dhabi, United Arab Emirates Email: mskhine@ecae.ac.ae

\begin{abstract}
Twitter as a microblogging platform often used to communicate each other in real time is a dominant practice of digital generation. Since its inception 10 years ago, the use of Twitter in educational settings has been growing considerably. Studies have been conducted to find out the affordances of Twitter and reported the advantages and drawbacks of the tool to use for teaching and learning in educational contexts. This study analyses research on Twitter as part of the doctoral dissertations in the universities in the USA. It identifies major categories and topics, describes the findings and discusses gaps in the research area from the educational technology perspective. The study selects 17 doctoral dissertations and examines the research aims, methodologies and results. The study proposes five overlapping categories. The analysis reveals that Twitter research in educational technology is still fragmented using diverse theories, and methodical advances are necessary for future research.
\end{abstract}

Keywords: educational technology; engagement; learning; professional development; review; social networks; twitter.

Reference to this paper should be made as follows: M.S. Khine (2017) 'Affordances of Twitter as a pedagogical tool in education: critical analysis of doctoral research studies', Int. J. Social Media and Interactive Learning Environments, Vol. 5, No. 1, pp.1-20.

Biographical note: Myint Swe Khine is a Professor and Chair of Assessment and Evaluation Centre at the Emirates College for Advanced Education, United Arab Emirates. He has taught at leading universities throughout Asia including Nanyang Technological University in Singapore and Curtin University in Australia. He holds Master's Degrees from the University of Southern California, USA, University of Surrey, and University of Leicester, UK and a Doctorate in Education from Curtin University in Australia. He is also an Adjunct Professor at Curtin University, Australia. He has taught graduate-level courses in assessment, evaluation and research design and methodology in education and supervised masters and doctoral students. His research interests are in learning sciences, instructional technology, science education and measurement and evaluation. He has published widely in international referred journals and edited several books. His recent publications include Noncognitive skills and factors in Educational Attainment (Senses Publishers, the Netherlands, 2016) and Visual-spatial ability in STEM Education: Transforming Research into Practice (Springer, Switzerland, 2017). 


\section{Introduction}

Since its introduction in 2006, Twitter is the most popular social networking platform with over 300 million monthly active users to date. With its share steadily growing, Twitter is virtually synonymous with and sets a standard as social networking platform. A message in Twitter is limited to 140 characters (known as a Tweet), and followers and general public can browse them on computers or mobile devices. It also provides prompt interaction among users regardless of the geographical locations and language differences. From the President to Prime Minister, Pope to priest and celebrities to commoners use Twitter to interact with their subjects and followers to reach out and convey important messages. Recent data show that $83 \%$ of the world's leaders are on Twitter. With such impressive numbers, educators and researchers alike are keen to explore its affordances and find ways to harness and apply in teaching and learning situations as a pedagogical tool (Rinaldo et al., 2011).

This study aimed to review the research related to Twitter conducted in the doctoral dissertations at the universities in the USA to discover the trends, focus and findings in relation to educational technology. The analysis from this study will broaden the view about Twitter, understand its role and gauge the potential applications in the educational contexts. It is the hope that the analysis can assist in charting the direction for future research.

\section{Review of research}

Twitter has been described as social networking platform, social media, microblogging tool and Web 2.0 application. The review article by Gao et al. (2012) draws together research carried out between 2008 and 2011 regarding the use of Twitter as microblogging tool. The authors critically analysed twenty-one studies on Twitter use in education in an attempt to answer:

1 What types of research have been published on microblogging in education?

2 How was microblogging used for teaching and learning in these studies?

3 What educational benefits did microblogging have on teaching and learning?

4 What suggestions and implications did the current research have for future microblogging research and practice?

The results show that in most studies, Twitter has been used to facilitate well-structured learning activities in educational settings. Some themes are also emerged in relation to learning community, participation and engagement, reflective thinking and collaborative learning. The analysis shows that most studies were conducted in limited duration, with a small number of participants and report the descriptive data. The studies identified major challenges including users' unfamiliarity with microblogging and lack of guidelines in participation. Some papers suggest that to be effective, teachers must make sure that such use is purposeful and relevant to students, set clear expectations and model effective Twitter use. The authors suggest that current studies concern with the use of Twitter as an extension or supplement formal classroom learning, and future research should consider both formal and informal learning in K-12 and higher education. The review also noted 
that most studies provide limited information about the participants and settings, implementation procedures and type of data collected and analysed. Future studies should incorporate methodological and scientific robustness in research design.

Carpenter and Krutka (2015) surveyed how and why educators use Twitter involving 755 K-16 educators in the US to better understand the role of Twitter plays in education. The study found that educators valued Twitter's personalised and immediate nature of communication that makes possible to form collaborative learning community. Some participants cited that Twitter breaks the isolation and creates new participatory culture and superior to traditional form of professional development. The authors suggested to explore ways to use Twitter in teacher professional development by exploiting the Twitter's affordances. Similarly, Visser et al. (2014) reported the use of Twitter as selfdirected professional development tool for K-12 teachers. An online survey with 324 teachers is used to determine their usage of, access to and perceptions of Twitter in education and training. The teachers perceived that they have formed meaningful relationships and personal learning networks and able to seek professional development for specific needs on Twitter platform. It was noted that for some teachers, Twitter use has been embedded in their routine that can improve the teaching practice.

According to Knight and Kaye (2016), social media such as Twitter provides new channel of communication and collaboration that can enhance students' learning experience. The authors surveyed 181 undergraduates, postgraduates and staff of the UK university in an attempt to find out general and academic usage of Twitter and student perception of usefulness of Twitter. The study found that there is a low uptake of Twitter in academic practices in the study context. The students perceived that providing details in practical issues are the most useful activity with Twitter. To answer the question of whether Twitter is an effective pedagogical tool in higher education, Bista (2015) conducted a study with 42 graduate students in a public university in the USA. The study involved setting up a course Twitter page and participants tweeted for 16 weeks. The students were also required to write a reflection paper at the end of the course. When the information from the reflection paper, course Tweet page and questionnaire was analysed, it was found that Twitter was a meaningful learning experience for all course participants. Although the participants used Twitter for the first time, they indicated the positive experiences of engaging in class discussions, group projects and other course related activities. In addition, participants noted that Twitter fostered active collaboration in the course; however, some noted that they do not envisage wider application of Twitter in schools or universities.

\section{Research questions}

This study investigates the use of online social networking site Twitter among educators in various educational settings by analysing the doctoral dissertations from the educational technology perspective. The following are the specific research questions.

1 What types of research have been conducted on Twitter in doctorial dissertations?

2 What are the affordances of Twitter as a pedagogical tool in education? 


\section{Research method}

\subsection{Searching and selection procedure}

The objective of the graduate education programs in most universities is to prepare the next generation of progressive scholars and educational leaders. Graduate students are expected to expand the knowledge of the disciplinary field by pursuing a unique topic of interest and make original contribution to the existing knowledge through vigorous research resulting in the doctoral dissertation (Lyons and Doueck, 2010). The analysis of doctoral dissertation research provides a useful method of assessing the intellectual growth of a particular field of research. In this study, doctoral dissertations related to Twitter are identified and explored the key areas and category of the studies, evaluated the methodology and synthesised the findings. It is hoped that this review will be useful for future attempts on Twitter research from educational technology perspective.

The focus of this paper is limited to the dissertations and theses submitted to the universities in the USA as partial fulfilment of the requirements for the doctoral degree. The search was conducted in three phases using ProQuest Dissertations and Theses database. This database is used due to its comprehensive collection of dissertations and theses, and the official digital dissertations archive for the Library of Congress and the database of record for graduate research (ProQuest, 2016). In the first phase, I made a basic search for the dissertations with the keyword Twitter in the last 5 years (2012-2016). In the second phase, further search is narrowed using the subject category: educational technology. More refinement was made in the third phase using Facebook as the title of the dissertation.

As of 10 October 2016, the basic search yielded 20,342 dissertations. ProQuest provides 100 subject categories to choose from including educational technology, higher education, educational psychology, educational sociology and instructional design. The search was narrowed by the subject term, educational technology, and 1328 dissertations were found in this category. Within this category, further refinement was made using the keyword Facebook in the title of the dissertations. This step filters the dissertations studies on general social networking sites. This refinement provided 23 dissertations. ProQuest covers dissertations in the universities in USA and Canada for both master and doctoral degrees. As this paper focuses on the doctoral dissertations in the USA universities, two dissertations at master level and three dissertations in Canadian universities were excluded in the analysis. Three more dissertations are also excluded since the contexts of the study do not directly relate to education domain.

\section{Analysis and results}

A total of 17 dissertations were identified, and after reading through the abstracts and based on the focus of the research, they are classified into five categories namely, concept learning, student engagement, language and literacy, personal learning networks and professional development (see Table 1). The details of the dissertations screened in this review are shown in Appendix A. 
Table 1 Classification of categories and topics

\begin{tabular}{ll}
\hline Category & Topics \\
\hline Concept learning (2) & Review tool for augmenting concept learning (Bissel, 2014) \\
Student engagement (3) & Distributed practice in concept learning (Buzzelli, 2014) \\
& Students' engagement and Twitter (Hirsh, 2012) \\
& Student engagement and academic performance (Yourechko, \\
& Twitter and student engagement and learning (Vohra, 2016) \\
Language and literacy (3) & Tool for English as a foreign language (Cheng, 2012) \\
& English language learners' literacy practice (Marissa, 2013) \\
& New literacies and teenage Twitter (Gleason, 2016) \\
Personal learning networks (4) & Personal learning network for professional development \\
& (Deyamport, 2013) \\
& Personal learning network for collaboration (Langhorst, 2015) \\
& Twitter's role in personal learning networks (Lowe, 2016) \\
& Use of Twitter for collaboration in professional learning \\
& communities (Pollard, 2015) \\
& Social capital building (Casey, 2013) \\
& Edchats discussions and professional development (Davis, \\
2012) & Principal professional development and Twitter (Sinanis, \\
2015) & Use of Twitter for online professional development (Strahler, \\
2014) & Twitter for professional development and lifelong learning \\
(Zellmer, 2014)
\end{tabular}

\subsection{Concept learning}

Concept learning has been defined as the search for and listing of attributes that can be used to distinguish exemplars from non-exemplars of various categories (Bruner et al., 1967). Out of 17 dissertations identified for this review, two dissertations focus on Twitter use for concept learning. Using Connectivist theory (Siemens, 2005), Bissel (2014) evaluated the concept learning in a marketing course with Twitter in her dissertation. The study utilised quasi-experimental method involving control and experimental groups with 41 students and used examination results, survey and interviews to triangulate the data. During the study period of eight weeks, the treatment group received daily tweets from the researcher features of the concepts and examples and non-examples to help students formulate the principles. The analysis of the examination results showed no significant differences between two groups, but the scores indicated that Twitter may function as an effective review tool to assist concept learning. Interview data revealed that student were engaged through reading and viewing images and graphs posted online. The students in the experimental group also found that the tweets from the professor are valuable, and they are generally pleased with Twitter integration in their study that enables them to read more, access further details and provide new perspectives. 
None of the students indicated that Twitter was a hindrance to the learning process. The researcher suggested that such study could be extended with larger number of participants and longer period of time.

The study by Buzzelli (2014) examined the effectiveness of Twitter for distributed practice in concept learning. Buzzelli believes that Twitter serves as an ideal tool for concept learning by sending tweets with examples and non-examples related to a specific concept. They conducted a study to examine if sending tweets would assist students in effectively learning concepts through distributed practice. The study involved 20 undergraduate students from an introductory USA history course in a university. The quantitative analysis found that Twitter could be equally effective as traditional review worksheet as a study tool. At the same time, it was found that simply incorporating twitter into a class does not create student engagement.

The concept learning involves a learning task in which the learner is trained to classify objects by being shown a set of examples. It refers to the human ability to learn categories for object and to recognise new instances of those categories. The research conducted by Bissel (2014) and Buzzelli (2014) shows that Twitter can be an effective to use as a review tool and useful for distributed practice in concept learning.

\subsection{Student engagement}

Hung and Khine (2006) suggested that if technology is used in a proper way, teachers can effectively engage the students in learning process. Sinatra et al. (2015) also noted that engagement is one of the important research topics in the field of educational psychology. Research shows that when students are engaged in their own learning, motivation and achievement can be increased. The study conducted by Junco et al. (2013) to assess Twitter use and outcomes of student collaboration, engagement and success showed that Twitter intervention promoted both student engagement and academic achievement. The authors noted that when students are required to use Twitter and faculty connects with them regularly, there is an increase in student engagement and grades.

Three dissertations focused on Twitter's effect on student engagement. Hirsh (2012) conducted an empirical study to explore the relationship of Twitter use to students' engagement and academic performance in online class. In this dissertation, the author examined the effect of Twitter usage as ungraded and non-formal engagement tool on academic performance as measured by course grade and engagement level with a group of 116 college students. The study employed quasi-experimental posttest design with control and treatment groups. The results showed that there was no statistically significant difference between two groups. But students in the treatment group self-reported that the tweets increased their level of engagement and also a higher final grade average. The survey results indicated that students benefited from the incorporation of Twitter in their learning. The author suggested that to use Twitter effectively in a course, students need to be clearly explained about the purpose of incorporating Twitter into the course and should view social networks as a digital corridor.

The study conducted by Yourechko (2016) explored the effect of Twitter in secondary student engagement and academic performance. This quantitative study involved 87 students randomly assigned to control and treatment groups. Although the control group participated in regularly scheduled classes, the treatment group was subject to using Twitter in the classroom during the 45 days of class sessions. The High School Survey of Student Engagement (HSSSE) was used as a basis to measure the potential change in 
student engagement. From the HSSSE survey, 29 items were selected to form a modified version to measure cognitive, intellectual and academic dimensions of student engagement. Students are encouraged to use Twitter as a mean of communication, and pretest and posttest were used to find out the effect. The results indicated that the effect of Twitter on student engagement found to be statistically significant, but there was no difference in academic performance.

In another study, Vohra (2016) explored how social presence on Twitter impacts student engagement and learning in a Grade 8 mathematics classroom during the 4-week long teaching session on data management concepts. The study involves 6 students and 1 classroom teacher at a public school. The data were collected using oral and written questionnaires, students' and teacher's reflective journals, students' and teacher's interviews, students' tweets and unit objectives and assessment. These sets of data were analysed through coding, categorising, determining themes and conducting a content analysis. A number of categories and themes were constructed from the teacher and students interviews. It was found that the teacher used Twitter to help students to learn mathematics in a variety of ways, including extending their learning by asking probing questions, posting challenging problems and providing feedback. The teacher and students believed that Twitter was a valuable tool to help improve the learning, because they were able to interact with each other outside of the classroom. The analysis also revealed that when the teacher integrated Twitter into mathematics instruction, student engagement was positively impacted because the social media helps create a positive learning environment. There were evidence that students collaborated on group assignments and interact with the teacher by answering probing questions and receiving feedback creating a smooth communication flow. The author concluded that Twitter use encourages students to learn concepts outside of the classroom, and Twitter allows students to form social bonding and relationships building to form community of learners.

The student engagement is a key area of concern for educators, and a number of strategies have been proposed and used in different educational settings to promote active learning. It is important that students pay attention and demonstrate curiosity and interest in the learning process. The results from the above-mentioned studies suggest that like many other technological tools, Twitter can be an effective medium engaging the students but not necessarily affects the academic achievement.

\subsection{Language and literacy}

The use of Twitter in language learning has been explored extensively by the researchers in the last few years. Some suggested that Twitter use can improve linguistic competence and help develop reading and writing skills (Cano, 2012; Stanley and Thornbury, 2013). Lomicka and Lord (2012) suggested that language teachers must consider whether Twitter is an activity that will bring benefits to the learners. They conducted an experiment to discover the role of Twitter in language learning involving 80 teachers in a graduate seminar course on language teaching methodology. The authors reported that a sense of community was established through the Twitter interactions beyond their classroom (Lord and Lomicka, 2014).

Greenhow and Gleason (2012) wrote that young people use Twitter in formal and non-formal settings creating interconnections, finding and conversing with friends, making new contacts, sharing information and user generated content. They noted that when learners and educators engage in social media such as Twitter, they may be 
developing new literacies. According to Davies (2012), new technologies may facilitate new social literacy practices through interactions among people that may not be possible by other means. After synthesising recent works, Greenhow and Gleason (2012) observed that students and teachers might benefit when Twitter is used for communication that allows asking and answering questions, focus or extend in-class discussions and generate information collaboratively. They suggested that tweeting practices of teachers and students may contribute to their development of new and old literacies.

Three dissertations in this category deals with Twitter as a tool for English as a foreign language (EFL), Twitter and English language learners' literacy practice and new literacies and teenage Twitter. The dissertation by Cheng (2012) explored the potential learning value of using Twitter as a tool for EFL reading and writing in a college in Taiwan. The study used quasi-experimental approach, and two equal groups consisting of 56 students were assigned as control and treatment groups. During two months of class sessions, both groups experienced the same learning materials and resources and teaching methods, except the control group engaged in free-writing activities whereas treatment used Twitter for major writing exercise. Both groups took pre- and posttests to assess the reading and writing skills. The data analysis indicates that different learning approaches did not have significant impact on students' reading and writing performance. But it was found that writing scores from pretest and posttest in each group were significantly different after the experiment. The study also used motivated strategy learning questionnaire (MSLQ) to measure the changes in learning attitudes after the experiment. It was found that Twitter-assisted learning had a significant positive influence on learning attitude.

In Marissa's (2013) study, literacy practices of two Indonesian college students were examined based on English texts that the students produced and interpreted on Twitter. The study focused on the practice of intertexuallity and explored how this practice relates to English language learner's formation of identity and negotiation to the development of English literacy. Ethnographic case study approach was used to collect data through in-depth interviews, observations and archive of online texts. The data obtained from the study were scientifically coded to ensure the validity and reliability. The author indicated the need to seek new ways to bridge students' out-of-school literacy practice and schoolbased literacy.

Gleason (2016) presented case studies that examined the Twitter practices of three teenagers over three years after collecting 60,000 tweets. The first study investigated new literacies practices with multimodel composition in Twitter, i.e. lifeblogging, mirroring and lifetweeting. These practices include visual mirroring and discursive mirroring and change of mirroring over time. The study found that these literacies were coconstructed, non-linear, multiple, social and action oriented. The second study investigated the relationship between new literacies practices and the identity work among teenage Twitter. The study also investigated how teenagers become feminists through new literacies. The third study explored how the use of Twitter in formal and informal learning can restructure the practices of teaching and learning, and may lead the pedagogy more humane. The study highlighted the issues related to young people's literacy, identity and learning practices with the use of Twitter.

Twitter as a communication medium is in the hands of citizens around the world and young and old are using in their everyday digital conversations. It will be an advantage for the educators if the medium can be harnessed for the benefits of the learners. The studies by Cheng (2012), Marissa (2013) and Gleason (2016) demonstrate that 
Twitter has significant positive impact on language learning and helps understand the literacy practices of the teenagers.

\subsection{Professional learning networks}

Holmes et al. (2013) noted that effective and continuous professional learning is vitally important for teachers in schools to make meaningful and sustainable changes to teacher practice. They studied the efficacy of Twitter as a medium for teachers to participate in professional learning network by analysing the tweets of the users. The study found that Twitter provides a channel to access new and relevant educational resources and allows participants to take control of their own learning. Similarly Trust et al. (2016) reported that many educators are using professional learning networks (PLN) for their professional development due to its advantages. When they analysed the survey data from 732 P-12 teachers on their experience on PLN, they found that such networks support professional growth of the teachers and able to address diverse interest and needs of the teachers.

Four dissertations focus on how Twitter can create personal PLN for teachers for professional development. In Deyamport's (2013) action research study involved eight elementary teachers who were trained to use Twitter to support the development of personal learning network. The study investigated how this Twitter-supported learning network is enhancing teachers' professional development. The data for this study were drawn from various sources that include participants' Twitter feeds, six weekly meeting, three focus group interviews, the researcher's journal and a survey questionnaire. The study specifically examined in what way the use of a Twitter supported personal learning network enhances teachers' personal professional development and how Twitter can be used to develop such network. The results supports that Twitter was found to be useful in developing a personal learning network, and all teachers in the study found that there is a value in Twitter use that supports the professional learning needs of teachers. But some teachers were passive participants reporting that there was not enough time to tweet, lack of interest in using Twitter and lack of confidence in tweeting. The researcher noted the length of time teachers used Twitter was not sufficient. It was found that participants in the study needed more than six weeks to learn how to use Twitter, and it requires more time to be comfortable using Twitter. The study also recommended to have the best practice sessions to model the use of resources, links and websites related to PLN.

The study by Langhorst (2015) explored the social studies teachers' use of Twitter and \#edchats for collaboration. The study focused on the weekly scheduled \#edchat tweets specifically designed for social studies teachers known as \#sschat which took place at a specific time. In an attempt to find out how social studies teachers are using the chat as a form of professional development, transcripts of 10 chat sessions with 290 participating individuals, and 2,821 separate tweets were coded and analysed. Once the emergent themes were identified, interviews were conducted with seven of the most consistent contributors from the chat sessions. The analysis showed the evidence of close personal connections among participants forming professional learning network consistent with phenomenon found in communities of practice. These specific chat sessions allow to narrow the content of the discussion specific to social studies. The participants in the chat sessions indicated that although adopting new technology is relatively easy, formal training on the methods of incorporating Twitter is lacking. This finding is consistent with other reports which highlight the need for training to help educators who feel less comfortable with new technologies. The study reiterated the need 
to provide assistant to teachers to enable them to collaborate and exchange views through Twitter in improving the instruction.

Lowe (2016) conducted a mixed methods study by collecting both quantitative and qualitative data to discover the role of Twitter in educational technology through an examination of the US educators and West Virginia (WV) educators. The study attempted to find out to what extent the US and WV educators are using Twitter to (i) employ instructional strategies, (ii) facilitate their own professional development, and (iii) create their own personal learning networks. In addition, the study also attempted to explore the barriers and challenges facing the US and WV educators when using Twitter professionally. The study collected three sets of data and the first set of data consisted of the extent of teachers beliefs on the use of Twitter for instructional strategies, professional development, personal learning networks and non-educational use. The second set of data consisted of content analysis of the participants' tweets. The third set is the qualitative data from semistructured interview with participants. The study found that fear is a large contributing factor why educators do not use Twitter professionally and instructional purpose. Teachers viewed that Twitter use for professional development is growing and teachers felt that those who use Twitter for their professional development should be given credit for their efforts. The study also found that the stronger the personal learning network use, the richer the professional development use. The participants in the study voiced the barriers and challenges in using Twitter in educational settings. The teachers felt that when new technology is used, the purpose must be made clear. The users need to be assured that technology is reliable and easy to adapt. Teachers will be hesitant to use technology if too much time is taken to learn, and technology is constantly changing. However, despite barriers and challenges, the researcher believed that Twitter could be a valuable technological tool for the educators.

According to Pollard (2015), the traditional means of professional development for teachers in the US are not meeting the needs of educators. But with the use of the social media networking tool such as Twitter, K-12 teachers can collaborate with each other to form PLN and communities. His dissertation explored how K-12 teachers are using Twitter for online professional learning community activities. The study utilised a qualitative approach with the use of interviews with 10 Twitter users and analysis of archives of the educational Twitter chats \#edchat and \#NT2T. Although \#edchat is a common platform and one of the most popular chats opens at specific time and it attracts educators from all around the globe, the New Teachers to Twitter (\#NT2T) is an open chat for teachers who are new to Twitter. The participants were asked why they choose to use Twitter for their professional development, what strategies they use and how they perceive their use is effective. In addition, the study seeks to explore how the teachers use Twitter for online professional development communities and how they personalise their experience. The study found that participants viewed educational Twitter chats are means to engage professional development activities, allow educators to develop online professional learning communities and able to personalise the professional development activities according to their own needs. The study recommends to explore among wider community of teachers and follow-up study to enhance the current findings. The study also suggests to explore other forms of social media including LinkedIn, Edmodo and Instagram.

Building communities of practice and PLN are key to professional growth. Exchanging, collaborating and sharing ideas, practices, materials and artefacts help teachers to learn and understand the current trends in their profession. Twitter seems to be 
an ideal medium for such activities. As reported by Deyamport (2013), Langhorst (2015) and Lowe (2016), Twitter can be an effective personal learning network for collaboration and professional development. According to Pollard (2015), Twitter can also provide opportunities for partnership and teamwork and form unique professional learning communities when the traditional means of professional development for teachers do not meet the needs in geographically diverse communities.

\subsection{Professional development}

Professional development is a major activity in teaching profession. There is no shortage of literature advocating the importance of and the need for continuing education and continuous professional development for all levels of teachers. In the age of internet and widespread availability of the web-based learning materials, professional development opportunities are shifting to online platforms where individuals can connect each other and form network of communities. Visser et al. (2014) noted that social networks contain information and resources that can be used by educators to develop professional practices. Technology today allows creation of personal learning networks to make interpersonal connections, engaged each other and share resources. When they made a study to explore how K-12 teachers use Twitter, the results showed that teachers highly value the platform as a mean of self-directed professional development. The study also found that Twitter allows to forge meaningful relationships among teachers to discuss and seek specific professional development needs.

By using social capital theory and six measures of social capital, Casey (2013) examined educators' use of Twitter by analysing the survey data obtained from 48 participants, qualitative interviews and 100 tweets from twelve participants for his dissertation. The research attempted to answer whether the use of Twitter by individual educators relates to social capital and whether such use relates more to bridging social or more to bonding social capital. In the first stage of the research, the researcher used a web-based survey adapted from internet social capital scales (ISCS) to collect data from PK-12 educators. In the second stage of the research, 12 individual telephone interviews were arranged to explore further on how the use of Twitter relates to social capital. The study also used tweets downloaded from the interview participants. This mixed method research allows collect both qualitative and quantitative data. The quantitative results indicate that participants' views related much to bridging social capital. Participants showed a stronger level of agreement on bridging social capital measures than bonding social capital. It was found that bridging social capital was more obvious in tweets than bonding social capital. This view is supported by the outcome of interviews with selected teachers. The study concluded that all participants showed high level of activity related to bridging social capital measures when building social capital online with the use of Twitter.

The dissertation by Davis (2012) investigated how K-12 public school teachers perceived the use of social network site such as Twitter for professional development. The research utilised qualitative embedded case study method that involved $17 \mathrm{~K}-12$ public school teachers across the US, from California to Wisconsin with teaching experience ranging from 1 to 36 years. The sample was drawn from the teachers who used \#edchat discussion at least twice a week over three consecutive months. The participants believed that subnetwork \#edchat allows collective inquiry, ask questions, provide feedback and engage in collaborative discussions. Some teachers also 
perceived that the platform provides sense of belonging and positive attitudes towards teaching. The teachers felt that \#edchat brings diverse perspectives and experiences and allows reflective thinking and benefited from the flow of information and ease of use. Some of the drawbacks include misunderstanding, misperception and not feeling heard. The author concluded that \#edchat served as a conduit in connecting teachers with community of practitioners, and there are benefits in using this platform for teachers' professional development.

Although most dissertations focus on students' and teachers' use of Twitter, Sinanis's (2015) dissertation deals with principal professional development in the US with the use of Twitter. The study attempted to include principals who are working in different levels of K-12 schools to represent diverse types of communities from different school districts throughout the country. Among the schools, two elementary principals, one middle school principal, two high school principals, and one K-12 principal were selected to involve in the study. The principals represented diversity in age, gender, years of experience and locations. The researcher conducted three interviews with each case study participants to discover their practices and goals for using Twitter through Google Hangout. The first interview focuses on the professional journey of the principals, and the second interview attempted to find out how they formed PLN and their interactions with the members in the group. In the final interview, the principals share artefact of their work context and ideas. The data from Twitter archive revealed how Twitter became a main source for their learning and establishing the professional learning network enriches their work as educational leaders. The study also noted that personal and professional developments are linked, and professional development must start on a personal level to be meaningful and sustainable. The participants' data confirmed that Twitter enable them to connect and establish professional relationship and are a valuable source for professional development.

Strahler (2014) examined how educators' uses and gratification expectancy of Twitter for professional development influences their perceived e-learning experience. The study also attempts to find out the demographics of participants who are looking for educational technology knowledge through Twitter. In his dissertation, Uses and Gratification Theory is employed to answer the research questions. A modified version of uses and gratification expectancy (UGE) questionnaire that comprised 22 statements was administered to measure four areas of cognitive, affective, personal integrative and social integrative aspects of UGE. The participants were identified in the \#edchat Twitter group, and online survey was conducted with the agreement of the respondents. Although the study targeted to 160 teachers, only 39 educators completed the entire survey. The sample represents wide geographical regions within the US, covering from Alaska to Wyoming, age ranges from under 22 to over 61 years, and majority $(76.9 \%)$ of the respondents were females. The results of the correlation analyses indicated that all four of the correlations were statistically significant and positively related to their perceived e-learning experience in which strong correlations were detected between cognitive UGE and personal UGE.

A subnetwork of \#edchat, \#IAedchat is designed to engage educators across the state of Iowa on variety of education topics and opens for discussion on every Sunday. In Zellmer's (2014) dissertation, he explored Iowa school leaders' perceptions of using \#IAedchat as a tool for professional development and lifelong learning. Among educators, receiving adequate and convenient professional development to meet the changing needs in performing their duties is the major concern. Social network sites such as Twitter 
provides opportunity to teachers and leaders of the schools to engage in meaningful and purposeful conversation through social interaction, shared personal experience and distributed knowledge. The researcher framed the research questions to better understand the phenomenon of Twitter use among educators. The specific research questions addressed in the dissertation were:

1 How Iowa school leaders use Twitter for professional development?

2 How they perceive participation in Twitter are contributing the online community of practice?

3 What are the benefits and shortcoming of Twitter as a means for professional development?

The participants selected in the study were 10 school administrators who actively participated in the past six weeks of the discussion for nine-month period. Interviews with participants were conducted through videoconferencing. From the analysis of the interviews, eight themes were emerged. They are networking, sharing knowledge and resources, starting and extending dialogue, sense of belonging, reflective thinking, inspiration, benefits and drawbacks of the chat. The educators perceived that \#IAedchat is easy to use and benefited in their professional learning. However, some teachers found it difficult to manage the flow of information and constraint in limited number of characters they can post.

Professional development activities for teachers take place in many forms. The most common activities are face-to-face taught courses, seminars and workshop at designated locations. But with the advances in technology and proliferation of digital devices, these activities can be conducted online asynchronously. An increasing number of professional development activities are taking place outside of the traditional settings. The study by Casey (2013) involves the use of Twitter for social capital building among teachers. The Twitter subnetwork \#edchat has been a popular platform for educators in exchanging ideas and forming learning communities. The studies by Davis (2012), Sinanis (2015), Strahler (2014) and Zellmer (2014) also explored the use of \#edchat and its variant @IAedchat as a tool for professional development of the teachers. The results show that educators perceived Twitter benefited in their professional development, but in some instances, the teachers reported the limitation of the technology and ease of use.

The aspects of the doctoral research, research purpose, context of the studies, data sources and research instruments, sample size and major findings are mapped in Appendix B.

\section{Discussion and conclusion}

This paper attempted to analyse the doctoral dissertations research on the use of Twitter as a pedagogical tool in education. Seventeen dissertations were screened from the ProQuest Dissertations and Theses Database. The paper identified educational use of Twitter in five major categories. These categories are concept learning, student engagement, language and literacy, personal learning networks and professional development. The paper then evaluated the methods used in the studies and examined the results. The dissertations referred to wide ranging theories from anthropology, psychology, sociology and technology domains including Connectivist theory (Siemens, 
2005), Uses and gratification theory (Katz et al., 1973) and Social capital theory (Putnam, 2001).

It was found that dissertation research on Twitter is largely exploratory with a limited number of subjects and experimental involving empirical measures. The statistical procedures employed in the studies were mostly descriptive and correlational analysis. The data were collected predominantly using self-reporting online surveys, remote interviews, and observations and analysis of tweets. Although these studies provide useful insight to the effects of Twitter in education, generalisations are not warranted. Future research should consider representative sampling techniques, increasing the sample size and inclusive in gender, ethnicity, demographics and cultural variables.

For educators and teachers, professional growth and development are important factors in their workplace. Professional development activities are increasingly organised in self-directed, self-selected and asynchronous online environments. Teachers need to be conversant with technology, in particular social networking platforms such as Twitter that allows to connect teachers from different regions and parts of the world to form community of practice. The studies in this paper demonstrated that teachers can use Twitter to seek and receive informal professional development in flexible ways to meet the individual needs and circumstances. Teachers can also use Twitter to communicate and engage with their students in effective ways. The implication is that teachers should develop a habit of mind in using social media tools to reach out to the students and community of practitioners, engage in meaningful conversations and advance the teaching and learning activities.

In the changing technological landscape, interconnectedness has been the key phenomena for citizens from all walks of life. Social networking becomes a common practice. Future research on Twitter will require methodological advances and sophistication to inform the stakeholders on Twitter effects and appropriate use as an educational tool. A robust content analysis and strong theoretical basis are critical to understand in depth of the affordances of Twitter. To further understand the role of Twitter and the connections that are created by the tool such as teacher-students relations and teacher-teacher relations should be further examined. The detailed and systematic analysis of Twitter exchanges may also shed light on how and why the connections are crucial to the users. Social network analysis and data mining techniques (Scott, 2011) could be employed to provide deeper understanding of Twitter as a pedagogical tool for educators.

\section{References}

Bista, K. (2015) 'Is Twitter an effective pedagogical tool in higher education? Perspectives of education graduate students', Journal of the Scholarship of Teaching and Learning, Vol. 15, No. 2, pp.83-102.

Bruner, J., Goodnow, J.J. and Austin, G.A. (1967) A Study of Thinking, Science Editions, New York.

Cano, E.V. (2012) 'Mobile learning with Twitter to improve linguistic competence at secondary schools', New Educational Review, Vol. 29, No. 3, pp.134-147.

Carpenter, J.P. and Krutka, D.G. (2015) 'Engagement through microblogging: educator professional development via Twitter', Professional Development in Education, Vol. 41, No. 4, pp.707-728. 
Davies, J. (2012) 'Facework on Facebook as a new literacy practice', Computers \& Education, Vol. 59, No. 1, pp.19-29.

Gao, F., Luo, T. and Zhang, K. (2012) 'Tweeting for learning: A critical analysis of research on microblogging in education published in 2008-2011', British Journal of Educational Technology, Vol. 43, No. 5, pp.783-801.

Greenhow, C. and Gleason, B. (2012) 'Twitteracy: Tweeting as a new literacy practice', The Educational Forum, Vol. 76, No. 4, pp.464-478.

Holmes, K., Preston, G., Shaw, K. and Buchanan, R. (2013) “"Follow” me: networked professional learning for teachers', Australian Journal of Teacher Education, Vol. 38, No. 12, pp.55-65.

Hung, D. and Khine, M.S. (Eds.) (2006) Engaged Learning with Emerging Technologies, Springer, Netherlands.

Junco, R., Elavsky, C.M. and Heiberger, G. (2013) 'Putting twitter to the test: Assessing outcomes for student collaboration, engagement and success', British Journal of Educational Technology, Vol. 44, No. 2, pp.273-287.

Katz, E., Blumler, J.G. and Gurevitch, M. (1973) 'Uses and gratifications research', The Public Opinion Quarterly, Vol. 37, No. 4, pp.509-523.

Knight, C.G. and Kaye, L.K. (2016) "“To tweet or not to tweet?"A comparison of academics' and students' usage of Twitter in academic contexts', Innovations in Education and Teaching International, Vol. 53, No. 2, pp.145-155.

Lomicka, L. and Lord, G. (2012) 'A tale of tweets: analyzing microblogging among language learners'. System, Vol. 40, No. 1, pp.48-63.

Lord, G. and Lomicka, L. (2014) 'Twitter as a tool to promote community among language teachers', Journal of Technology and Teacher Education, Vol. 22, No. 2, pp.187-212.

Lyons, P. and Doueck, H.J. (2010) The Dissertation: From Beginning to End, Oxford University Press, Oxford.

Putnam, R. (2001) 'Social capital: measurement and consequences', Canadian Journal of Policy Research, Vol. 2, No. 1, pp.41-51.

ProQuest (2016) Dissertations and Theses [online]. Available at: http://www.proquest.com/ products-services/dissertations/ (access 10 October 2016).

Rinaldo, S.B., Tapp, S. and Laverie, D.A. (2011) 'Learning by tweeting: Using Twitter as a pedagogical tool', Journal of Marketing Education, Vol. 33, No. 2, pp.193-203.

Scott, J. (2011) 'Social network analysis: developments, advances, and prospects', Social network analysis and mining, Vol. 1, No. 1, pp.21-26.

Siemens, G. (2005) 'Connectivism: learning as network-creation', ASTD Learning News, Vol. 10, No. 1.

Sinatra, G.M., Heddy, B.C. and Lombardi, D. (2015) 'The challenges of defining and measuring student engagement in science', Educational Psychologist, Vol. 50, No. 1, pp.1-13.

Stanley, G. and Thornbury, S. (2013) Language Learning with Technology: Ideas for Integrating Technology in the Classroom. Cambridge University Press.

Trust, T., Krutka, D.G. and Carpenter, J.P. (2016) “"Together we are better”: professional learning networks for teachers', Computers \& Education, Vol. 102, pp.15-34.

Visser, R.D., Evering, L.C. and Barrett, D.E. (2014) 'Twitter for teachers: the implications of Twitter as a self-directed professional development tool for K-12 teachers', Journal of Research on Technology in Education, Vol. 46, No. 4, pp.396-413. 


\section{Appendix A}

List of dissertations screened in the Review*

Bissell, J. (2014). Evaluation of student achievement on course concepts by augmenting content through Twitter. (Order No. 3648352, Robert Morris University).

Buzzelli, A. Twitter in the Classroom: Determining the Effectiveness of Utilizing a Microblog for Distributed Practice in Concept Learning. (Order No. 3648353, Robert Morris University).

Casey, B. (2013). Building social capital online: Educators' uses of Twitter. (Order No. 3564480 , Edgewood College).

Cheng, H.Y. (2012). Applying Twitter to EFL reading and writing in a Taiwanese college setting. (Order No. 3539349. Indiana State University).

Davis, K. (2012). Learning in 140 Characters: Teachers' Perceptions of Twitter for Professional Development. (Oder No. 3531637, University of Phoenix).

Deyamport, W. (2013). An implementation of a Twitter-supported personal learning network to individualize teacher professional development. (Order No. 3559871, Capella University).

Gleason, B. (2016). The world of teenage Twitter: New literacies, identity work, and humanizing pedagogy. (Order No. 10144339, Michigan State University).

Hirsh, O. (2012). The Relationship of Twitter Use to Students' Engagement and Academic Performance in Online Classes at an Urban Community College. (Order No. 3545578. Walden University).

Langhorst, E. (2015). Social Studies Teachers' Use of Twitter and \#edchats for Collaboration. (Order No. 3700962, Walden University).

Lowe, R. (2016). Education all a 'Twitter: Twitter's role in educational technology. (Order No. 10125269, Marshall University).

Marissa, D. (2013). Intertextuality, identity works, and second language literacy development in the digital media: An ethnographic case study of two Indonesian college students' literacy practice on Twitter. (Order No. 3611936, University of Maryland).

Pollard, J. (2015). The use of Twitter as a collaborative environment for K-12 teachers: Perceptions of 140 character professional learning communities. (Order No. 10015220, Wilkes University).

Sinanis, A. (2015). Principal professional development and Twitter: 140 characters of learning. (Order No. 3721070, University of Pennsylvania).

Strahler, D. (2014). Educators' perceptions of twitter for educational technology professional development: A uses and gratifications expectancy model. (Order No. 3646860, Duquesne University).

Vohra, S. (2016). How Social Presence on Twitter Impacts Student Engagement and Learning in a Grade 8 Mathematics Classroom. (Order No. 10123835, Walden University).

Yourechko, A. (2016). The Effect of Twitter on Secondary Student Engagement and Academic Performance. (Order No. 10126826, Wilkes University).

Zellmer, A. (2014). School leaders' perceptions of using \#iaedchat: A subnetwork of Twitter for professional development and lifelong learning. (Order No. 3685709, University of South Dakota).

*All dissertations are retrieved from ProQuest Dissertations and Theses Database. 
Appendix B Mapping dissertations on Twitter

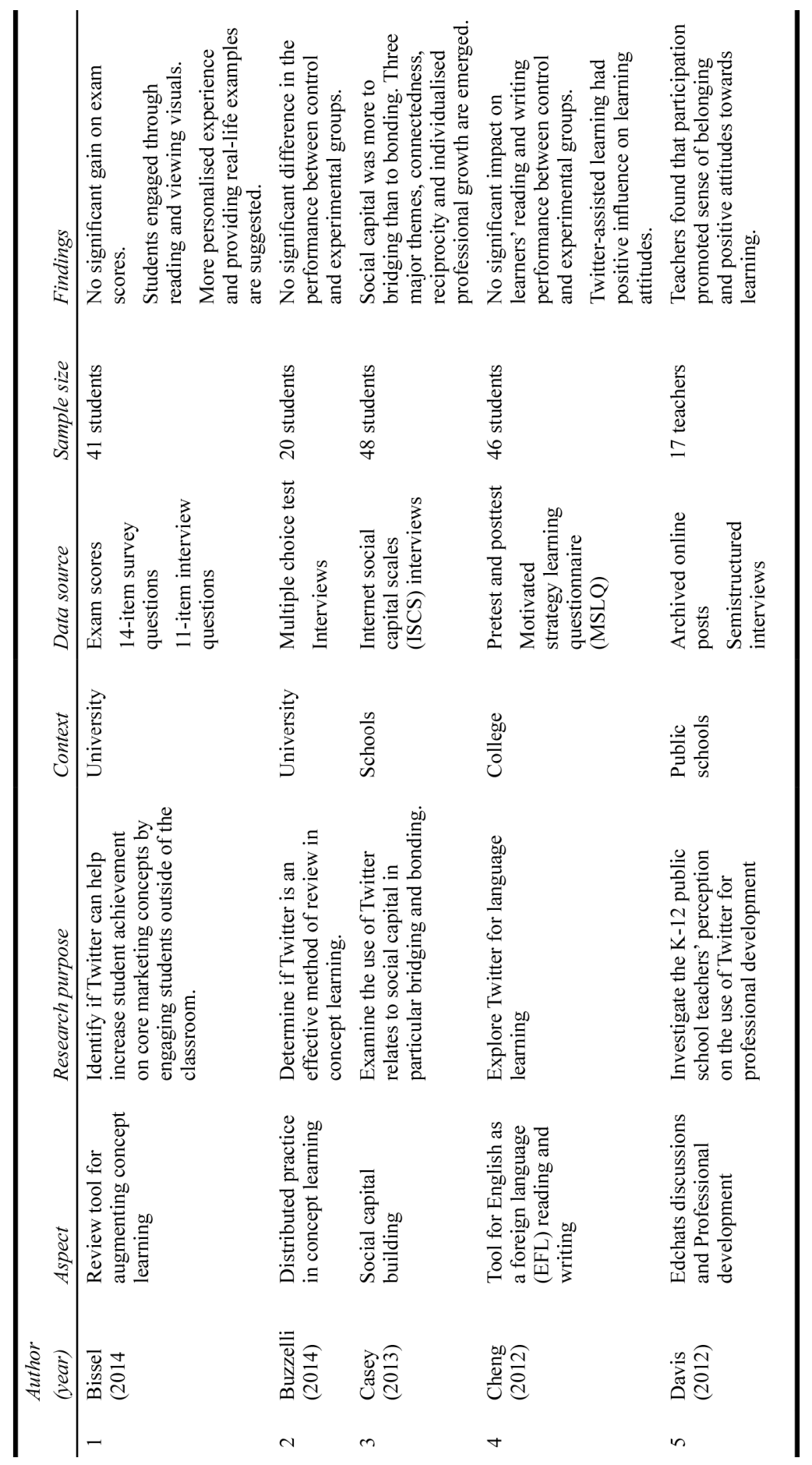




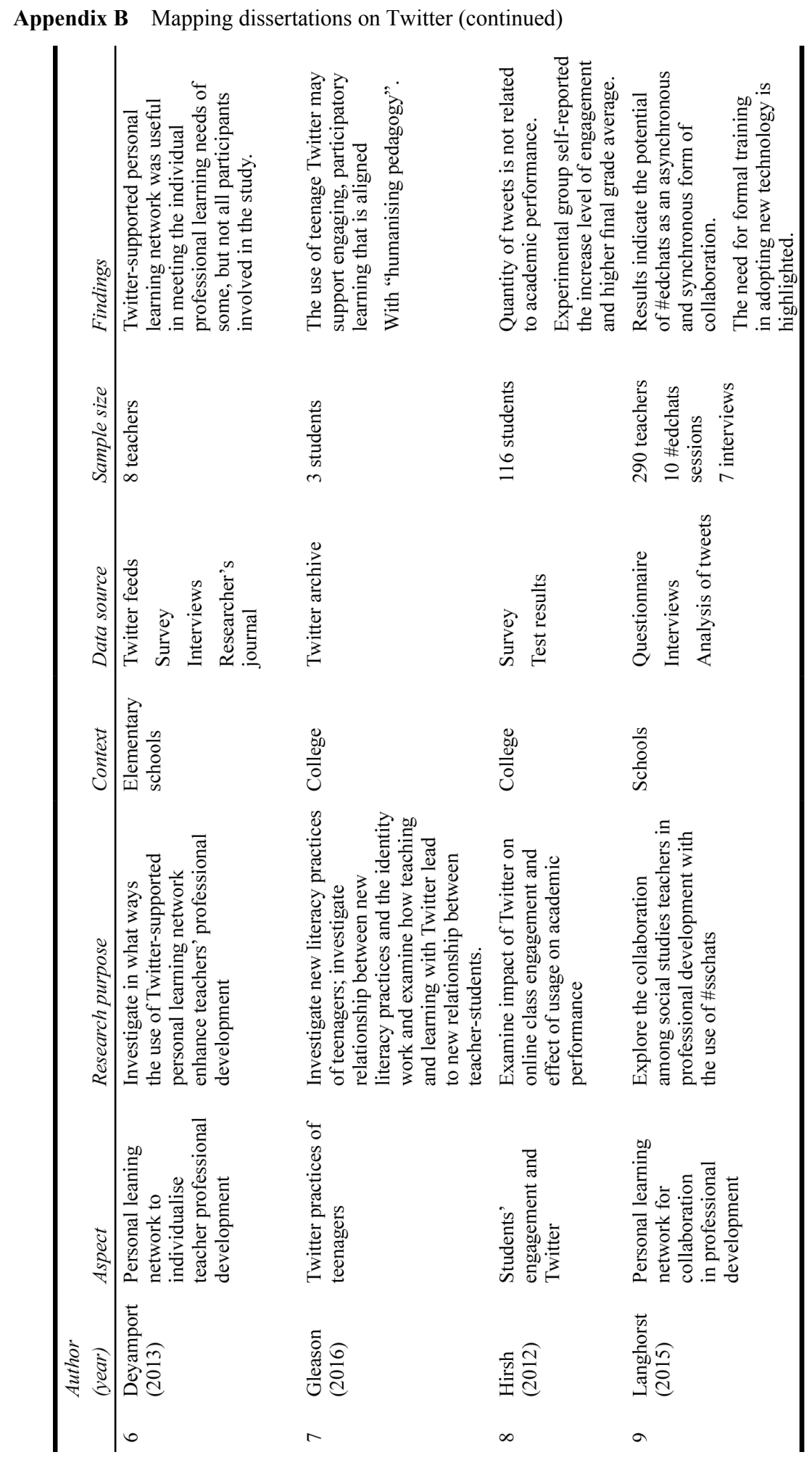


Appendix B Mapping dissertations on Twitter (continued)

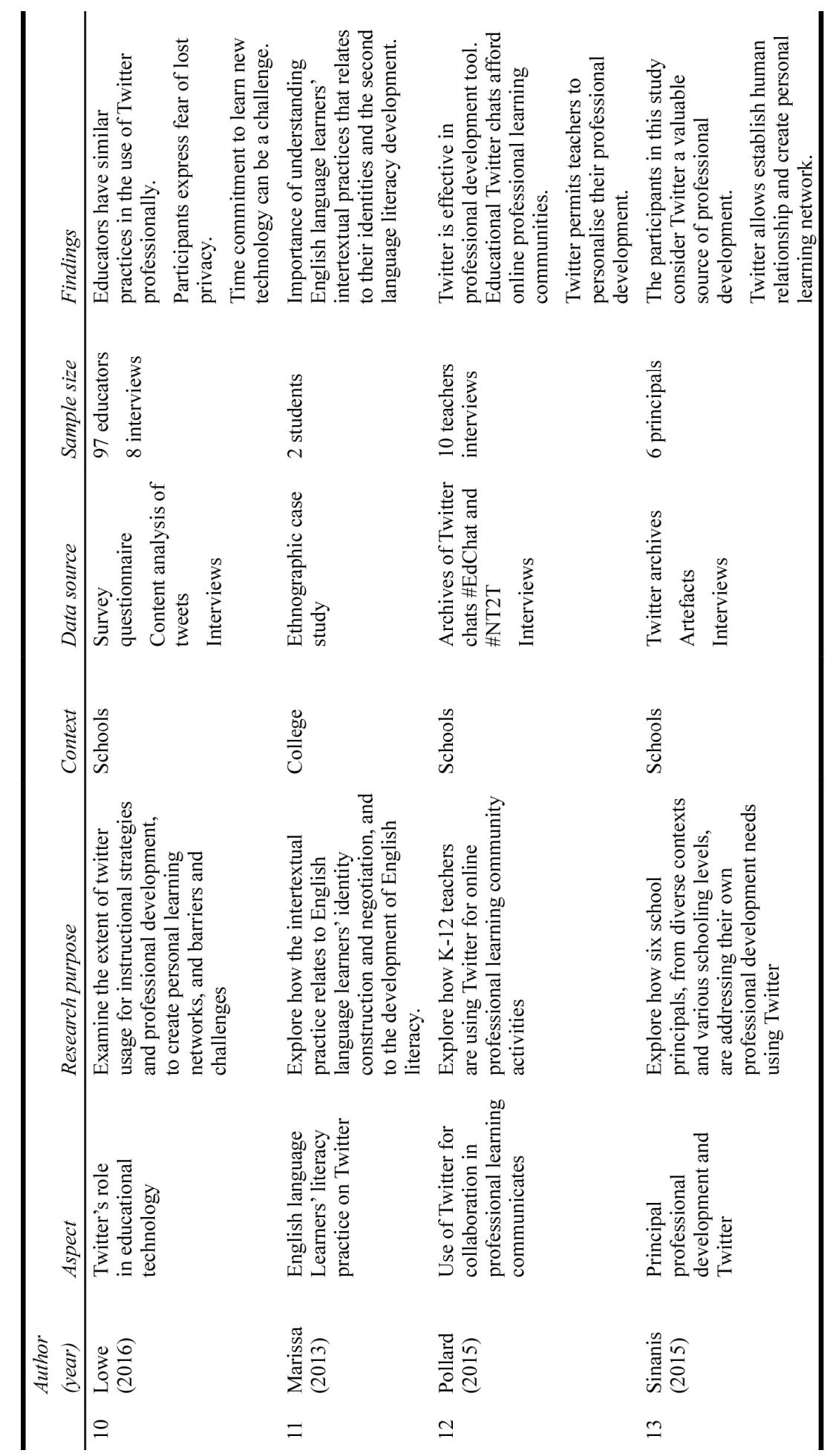


Appendix B Mapping dissertations on Twitter (continued)

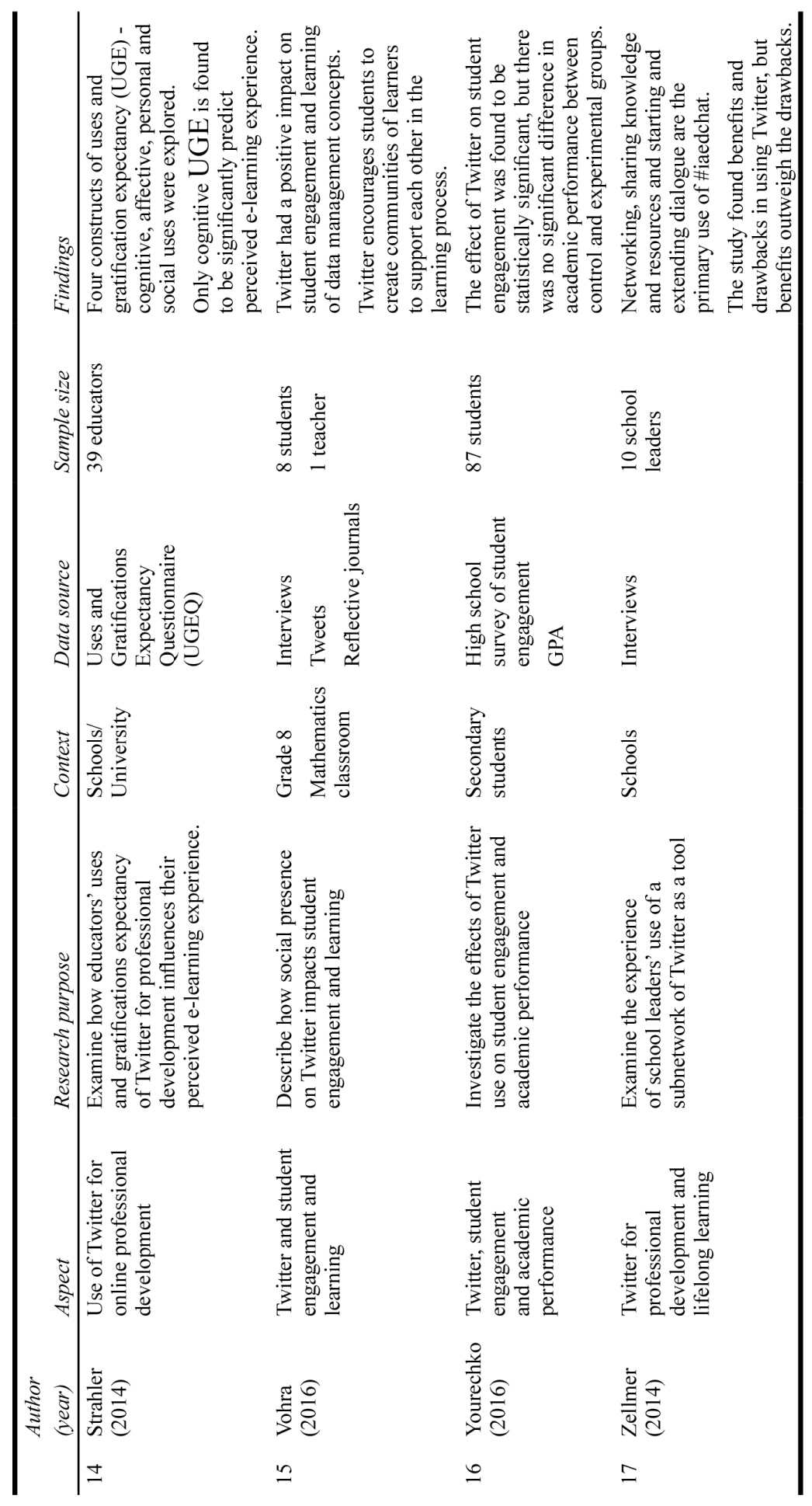

